\title{
The Least Square Nucleolus is a Normalized Banzhaf Value
}

\author{
J.M. Alonso-Meijide ${ }^{1}$, M. Álvarez-Mozos ${ }^{2}$, and M.G. Fiestras-Janeiro ${ }^{3}$ \\ ${ }^{1}$ Departamento de Estatística e Investigación Operativa, Universidade de Santiago de \\ Compostela, Spain. \\ ${ }^{2}$ Departament de Matemàtica Econòmica, Financera i Actuarial, Universitat de Barcelona, \\ Spain. \\ ${ }^{3}$ Departamento de Estatística e Investigación Operativa, Universidade de Vigo, Spain.
}

\begin{abstract}
In this note we study a truncated additive normalization of the Banzhaf value. We are able to show that it corresponds to the Least Square nucleolus (LS-nucleolus), which was originally introduced as the solution of a constrained optimization problem (Ruiz et al., 1996). Thus, the main result provides an explicit expression that eases the computation and contributes to the understanding of the LS-nucleolus. Lastly, the result is extended to the broader family of Individually Rational Least Square values (Ruiz et al., 1998b).
\end{abstract}

Keywords: Coalitional games; Banzhaf value; Efficiency; Least square nucleolus

A cooperative game with transferable utility (just TU game from now on) is a pair $(N, v)$ where $N$ is a finite set of players and $v$, the characteristic function, is a real valued function on $2^{N}$ with $v(\emptyset)=0$. Consider a TU game $(N, v)$. A vector $x \in \mathbb{R}^{N}$ is called an allocation. For each allocation $x \in \mathbb{R}^{N}$ and each nonempty coalition $S \subseteq N$, the excess of coalition $S$ at allocation $x$ is given by $e(S, x)=v(S)-x(S)$ with $x(S)=\sum_{i \in S} x_{i}$. The average excess at $x$ is given by $\bar{e}(v)=\frac{1}{2^{n}-1} \sum_{\emptyset \neq S \subset N} e(S, x)$. An allocation, $x$, is efficient whenever $x(N)=v(N)$. The set of imputations of $(N, v)$ is defined as the set of all efficient allocations that are individually rational, i.e., $I(N, v)=\left\{x \in \mathbb{R}^{N}: x(N)=\right.$ $v(N), x_{i} \geq v(\{i\})$, for every $\left.i \in N\right\}$. We denote by $\mathcal{G}$ the set of all TU games with a nonempty imputation set. Let $\mathcal{H} \subseteq \mathcal{G}$, a value on $\mathcal{H}$ is a map, $\mathrm{f}$, that associates an allocation to every TU game in $\mathcal{H}$, i.e., for every $(N, v) \in \mathcal{H}, \mathrm{f}(N, v) \in \mathbb{R}^{N}$. One of these values is the Banzhaf value (Owen, 1975) that we denote by B. For every $(N, v) \in \mathcal{G}$ and $i \in N$, the Banzhaf value is defined by

$$
\mathrm{B}_{i}(N, v)=\frac{1}{2^{n-1}} \sum_{S \subseteq N \backslash i}(v(S \cup i)-v(S)) .^{1}
$$

\footnotetext{
${ }^{1}$ We will write $S \cup i$ instead $S \cup\{i\}$ and $S \backslash i$ instead $S \backslash\{i\}$ to simplify the notation. Given a finite set $S$, we denote by lowercase $s$ its number of elements.
} 
It is well known that the Banzhaf value is not, in general, an efficient allocation. Hammer and Holzman (1992) proposed two efficient values based on the Banzhaf value that we call the multiplicative normalization of the Banzhaf value and the additive normalization of the Banzhaf value. Let $\mathcal{H} \subseteq \mathcal{G}$ be the set of TU games satisfying $\sum_{j \in N} \mathrm{~B}_{j}(N, v) \neq 0$. The multiplicative normalization of the Banzhaf value, $\mathrm{B}^{\mathrm{m}}$, assigns to every $(N, v) \in \mathcal{H}$ an allocation which is proportional to $\mathrm{B}(N, v)$. Formally, $\mathrm{B}^{\mathrm{m}}$ is the value on $\mathcal{H}$ defined for every $(N, v) \in \mathcal{H}$ and $i \in N$ by

$$
\mathrm{B}_{i}^{\mathrm{m}}(N, v)=\frac{v(N)}{\sum_{j \in N} \mathrm{~B}_{j}(N, v)} \mathrm{B}_{i}(N, v) .
$$

Given a TU game, the additive normalization of the Banzhaf value, $\mathrm{B}^{\mathrm{a}}$, is obtained by adding the same amount to every agent's Banzhaf value. Formally, $B^{a}$ is the value on $\mathcal{G}$ defined for every $(N, v) \in \mathcal{G}$ and $i \in N$ by

$$
\mathrm{B}_{i}^{\mathrm{a}}(N, v)=\mathrm{B}_{i}(N, v)+\frac{1}{n}\left(v(N)-\sum_{j \in N} \mathrm{~B}_{j}(N, v)\right) .
$$

Ruiz et al. (1996) proved the equivalence between the additive normalization of the Banzhaf value and the Least Square prenucleolus, LS-prenucleolus. The LS-prenucleolus is defined for every $(N, v) \in \mathcal{G}$ as the optimal solution of the optimization problem

$$
\begin{aligned}
& \min \sum_{\emptyset \neq S \subseteq N}(e(S, x)-\bar{e}(v))^{2} \\
& \text { s.t. } x(N)=v(N) .
\end{aligned}
$$

It is clear that, in general, the optimal solution of this problem is not an imputation. In order to solve this drawback, Ruiz et al. (1996) defined the Least Square nucleolus, LS-nucleolus, for every $(N, v) \in \mathcal{G}$ as the optimal solution of the optimization problem

$$
\begin{array}{ll}
\min & \sum_{\emptyset \neq S \subseteq N}(e(S, x)-\bar{e}(v))^{2} \\
\text { s.t. } & x(N)=v(N) \\
& x_{i} \geq v(\{i\}), \text { for every } i \in N .
\end{array}
$$

Since both the Banzhaf value and the LS-nucleolus satisfy strategic equivalence ${ }^{2}$ from now on we assume that for every $i \in N, v(\{i\})=0$. If the LS-prenucleolus is an imputation, it coincides with the LS-nucleolus, but in general, both concepts provide different allocations. Ruiz et al. (1996) proposed the following algorithm to obtain the LS-nucleolus.

\section{Algorithm 1.}

\footnotetext{
${ }^{2} \mathrm{~A}$ value on $\mathcal{G}, \mathrm{f}$, satisfies strategic equivalence if for every $(N, v) \in \mathcal{G}, \alpha>0$, and $\beta \in \mathbb{R}^{N}, \mathrm{f}(N, \alpha v+$ $\beta)=\alpha \mathrm{f}(N, v)+\beta$, where $(N, \alpha v+\beta)$ is defined for every $S \subseteq N$ by $(\alpha v+\beta)(S)=\alpha v(S)+\beta(S)$.
} 
Step 1. Take $k=1, x^{1}=\mathrm{B}^{\mathrm{a}}(N, v)$ and $M^{1}=\left\{i \in N: x_{i}^{1}(N, v)<0\right\}$.

Step 2. Take $k=k+1$. For every $j \in N$,

$$
\begin{aligned}
& \qquad x_{j}^{k}= \begin{cases}x_{j}^{k-1}+\frac{x^{k-1}\left(M^{k-1}\right)}{n-m^{k-1}} & \text { if } j \notin M^{k-1} \\
0 & \text { otherwise } 0\end{cases} \\
& \text { and } M^{k}=M^{k-1} \cup\left\{i \in N: x_{i}^{k}(N, v)<0\right\} .
\end{aligned}
$$

Step 3. If $M^{k}=M^{k-1}, x^{k}$ is the LS-nucleolus. Otherwise, go to Step 2.

In this note we prove that the LS-nucleolus of a TU game with a non-empty imputation set is also a normalization of the Banzhaf value. The truncated normalization of the Banzhaf value, $\mathrm{B}^{\mathrm{t}}$, is the value on $\mathcal{G}$ defined for every $(N, v) \in \mathcal{G}$ as follows:

1. If for every $l \in N,\left(\sum_{j \in N} \mathrm{~B}_{j}(N, v)-v(N)\right) / n \leq \mathrm{B}_{l}(N, v)$, then for every $i \in N$,

$$
\mathrm{B}_{i}^{\mathrm{t}}(N, v)=\mathrm{B}_{i}(N, v)+\frac{1}{n}\left(v(N)-\sum_{j \in N} \mathrm{~B}_{j}(N, v)\right) .
$$

2. If there is some $l \in N$ with $\left(\sum_{j \in N} \mathrm{~B}_{j}(N, v)-v(N)\right) / n>\mathrm{B}_{l}(N, v)$. Then, for every $i \in N$,

$$
\mathrm{B}_{i}^{\mathrm{t}}(N, v)=\mathrm{B}_{i}(N, v)-\min \left\{\mathrm{B}_{i}(N, v), c\right\}
$$

where $c \geq 0$ such that $\sum_{i \in N} \min \left\{\mathrm{B}_{i}(N, v), c\right\}=\sum_{j \in N} \mathrm{~B}_{j}(N, v)-v(N)$.

The truncated normalization of the Banzhaf value above emerged while looking for an additive normalization of the Banzhaf value that satisfies individual rationality. The two cases considered distinguish games where $B^{a}$ satisfies individual rationality and games where it fails to do so. In the first case $B^{t}$ selects the allocation given by $B^{a}$. In the second case, there is some player with a negative payoff according to $B^{a}$. In this case, the payoffs according to the Banzhaf value are reduced in a fixed amount subject to no one receiving a negative payoff. The solution in this second case is inspired by the CEL bankruptcy rule (Aumann and Maschler, 1985).

Next we show that the truncated normalization of the Banzhaf value is, in fact, the LS-nucleolus.

Proposition 1. For every $(N, v) \in \mathcal{G}$, the allocation given by the truncated normalization of the Banzhaf value is the LS-nucleolus of $(N, v)$.

Proof. Take $A=\left\{i \in N:\left(\sum_{j \in N} \mathrm{~B}_{j}(N, v)-v(N)\right) / n \leq \mathrm{B}_{i}(N, v)\right\}$. If $A=N$ then $\mathrm{B}^{\mathrm{t}}(N, v)=\mathrm{B}^{\mathrm{a}}(N, v)$ and it is an imputation. Then, by Ruiz et al. (1996) it is the LSnucleolus. Now, assume that there is some $l \in N$ with $\left(\sum_{j \in N} \mathrm{~B}_{j}(N, v)-v(N)\right) / n>$ $\mathrm{B}_{l}(N, v)$. We show that Algorithm 1 ends up at allocation $\mathrm{B}^{\mathrm{t}}(N, v)$. Take $k=1$, $x^{1}=\mathrm{B}^{\mathrm{a}}(N, v)$, and $M^{1}=\left\{i \in N: x_{i}^{1}(N, v)<0\right\}$. Then, $M^{1} \neq \emptyset$. Besides, $M^{1} \neq N$ 
because the imputation set $I(N, v) \neq \emptyset$ and $v(N) \geq 0$. Take $k=2$ and we obtain $x^{2}$ following Step 2 in Algorithm 1. Then, $x_{j}^{2}=0$, for every $j \in M^{1}$ and

$$
x_{j}^{2}=x_{j}^{1}+\frac{x^{1}\left(M^{1}\right)}{n-m^{1}}=\mathrm{B}_{j}^{\mathrm{a}}(N, v)+\frac{1}{n-m^{1}} \sum_{i \in M^{1}} \mathrm{~B}_{i}^{\mathrm{a}}(N, v),
$$

for every $j \notin M^{1}$. Taking into account that

$$
\begin{aligned}
\frac{1}{n-m^{1}} \sum_{i \in M^{1}} \mathrm{~B}_{i}^{\mathrm{a}}(N, v) & =\frac{1}{n-m^{1}} \sum_{i \in M^{1}} \mathrm{~B}_{i}(N, v)+\frac{m^{1}}{\left(n-m^{1}\right) n}\left(v(N)-\sum_{i \in N} \mathrm{~B}_{i}(N, v)\right) \\
& =\frac{m^{1}}{n\left(n-m^{1}\right)}\left(v(N)-\sum_{i \in N \backslash M^{1}} \mathrm{~B}_{i}(N, v)\right)+\frac{n-m^{1}}{n\left(n-m^{1}\right)} \sum_{i \in M^{1}} \mathrm{~B}_{i}(N, v),
\end{aligned}
$$

we obtain

$$
\begin{aligned}
x_{j}^{2} & =\mathrm{B}_{j}(N, v)+\frac{1}{n}\left(v(N)-\sum_{i \in N} \mathrm{~B}_{i}(N, v)\right)+\frac{m^{1}}{n\left(n-m^{1}\right)}\left(v(N)-\sum_{i \in N \backslash M^{1}} \mathrm{~B}_{i}(N, v)\right) \\
& +\frac{n-m^{1}}{n\left(n-m^{1}\right)} \sum_{i \in M^{1}} \mathrm{~B}_{i}(N, v)=\mathrm{B}_{j}(N, v)+\frac{1}{n-m^{1}}\left(v(N)-\sum_{i \in N \backslash M^{1}} \mathrm{~B}_{i}(N, v)\right) .
\end{aligned}
$$

Next, $M^{2}=\left\{j \in N: x_{j}^{2}<0\right\}$. If $M^{2}=M^{1}$, we finish. Otherwise, we repeat Step 2 in Algorithm 1. In the end we obtain $x_{j}^{k}=0$, for every $j \in M^{k-1}$ and

$$
x_{j}^{k}=\mathrm{B}_{j}(N, v)+\frac{1}{n-m^{k-1}}\left(v(N)-\sum_{i \in N \backslash M^{k-1}} \mathrm{~B}_{i}(N, v)\right)
$$

for every $j \in N \backslash M^{k-1}$, where $M^{k-1}=\left\{j \in N: x_{j}^{k-1}<0\right\}$. This allocation is precisely $\mathrm{B}^{\mathrm{t}}(N, v)$.

Let us examine the LS-nucleolus in some examples. The first two are instances of weighted majority games. A weighted majority game is determined by a weight $w_{i} \geq 0$ for every player $i \in N$ and a quota $q>0$ that determines the minimum joint weight that a coalition must reach in order to be a winning coalition. The worth of a coalition is 1 if it is winning and 0 otherwise. We denote a weighted majority game by $\left[q ; w_{1}, \ldots, w_{n}\right]$.

Example 1. Let $(N, v)$ be the weighted majority game with $N=\{1,2,3,4,5\}$ and $[5 ; 3,2,2,2,1]$. Its Banzhaf value is $\mathrm{B}(N, v)=(0.5675,0.3125,0.3125,0.3125,0.1875)$. Then, $\sum_{i \in N} \mathrm{~B}_{i}(N, v)=1.6875>v(N)=1$. Thus, $\mathrm{B}^{\mathrm{t}}(N, v)=(0.425,0.175,0.175,0.175$, $0.05)=\mathrm{B}^{\mathrm{a}}(N, v)$. In this case each player's Banzhaf value is reduced in 0.1375.

Example 2. Let $(N, v)$ be the weighted majority game with $N=\{1,2,3,4\}$ and $[3 ; 1,1,1,0]$. It easy to check that $\mathrm{B}(N, v)=(0.25,0.25,0.25,0)$. Then, $\sum_{i \in N} \mathrm{~B}_{i}(N, v)=$ $0.75<v(N)=1$. Thus, $\mathrm{B}^{\mathrm{t}}(N, v)=(0.3125,0.3125,0.3125,0.1875)=\mathrm{B}^{\mathrm{a}}(N, v)$. In this case the Banzhaf value of every player can be increased in 0.0625 , keeping individual rationality. Although player 4 is a null player, he receives the minimum amount. 
Lastly, we revisit the example given in Ruiz et al. (1996).

Example 3. Let $(N, v)$ be the $T U$ game given by $N=\{1,2,3,4,5\}$ and the characteristic function defined as $v(3,4)=1, v(3,5)=1, v(4,5)=1, v(1,3,4)=1, v(1,3,5)=$ $1, v(1,4,5)=1, v(2,3,4)=1.4, v(2,3,5)=1, v(2,4,5)=1, v(3,4,5)=1.75$, $v(1,2,3,4)=1.75, v(1,2,3,5)=1, v(1,2,4,5)=1, v(1,3,4,5)=2, v(2,3,4,5)=2$, $v(N)=2$. The characteristic function $v$ assigns 0 to all the remaining coalitions. Then,

$$
\begin{aligned}
& \mathrm{B}(N, v)=(0.0375,0.0875,0.80625,0.80625,0.6625), \\
& \mathrm{B}^{\mathrm{a}}(N, v)=(-0.0425,0.0075,0.72625,0.7265,0.5825), \text { and } \\
& \mathrm{B}^{\mathrm{t}}(N, v) \approx(0,0,0.714583,0.714583,0.570833) .
\end{aligned}
$$

According to the Banzhaf value, we observe that there are two weak (but not symmetric) players, 1 and 2, and three strong players, 3, 4, and 5. In this case, the additive normalization of the Banzhaf value proposes a payoff below the first agent's standalone worth. Consequently, $\mathrm{B}^{\mathrm{t}}$ differs from $\mathrm{B}^{\mathrm{a}}$. Note that the truncation takes place not only on the payoff of player 1 but also on the payoff of player 2 , who get both 0 .

Next, we briefly describe how the above procedure can be generalized to the class of individually rational least square values (Ruiz et al., 1998a). A map $w$ from $\{1, \ldots, n-1\}$ on $\mathbb{R}_{+}$is called a symmetric weight function. This map is used to weight the excess vector at efficient allocations. Coalition $N$ is excluded because $e(N, x)=0$ for every efficient allocation $x$. Besides, all coalitions of the same size have the same weight. Take a symmetric weight function $w$. The $I R L S^{w}$ value is defined for every $(N, v) \in \mathcal{G}$ as the optimal solution of the optimization problem

$$
\begin{array}{ll}
\min & \sum_{\emptyset \neq S \subseteq N}(e(S, x)-\bar{e}(v))^{2} w(s) \\
\text { s.t. } & x(N)=v(N) \\
& x_{i} \geq v(\{i\}), \text { for every } i \in N .
\end{array}
$$

If we take $w(s)=1$, for every $s=1, \ldots, n-1$, the $I R L S^{w}$ value of the TU game $(N, v)$ coincides with its LS-nucleolus. In case the feasible region of this optimization problem is only the set of efficient allocations, the optimal solution is called a least square value, $L S^{w}$. Ruiz et al. (1998a) proposed a procedure to obtain the IRLS $S^{w}$ value quite similar to Algorithm 1. In fact, both procedures differ only in the starting point, being $L S^{w}(N, v)$ the initial allocation used to obtain the $I R L S^{w}$ value.

Ruiz et al. (1998a) show that for every $(N, v) \in \mathcal{G}$ the allocation $L S^{w}(N, v)$ is given by

$$
L S_{i}^{w}(N, v)=\frac{v(N)}{n}+\frac{1}{\alpha n}\left(n a_{i}(v)-\sum_{j \in N} a_{j}(v)\right)
$$

where $a_{i}(v)=\sum_{S \ni i} v(S) w(s)$, for every $i \in N$ and

$$
\alpha=\sum_{s=1}^{n-1} w(s)\left(\begin{array}{c}
n-2 \\
s-1
\end{array}\right)
$$


It is easy to check, after some reorganization of the terms in Expression (3), that

$$
L S_{i}^{w}(N, v)=\frac{1}{\alpha} \sum_{S \ni i}(v(S)-v(S \backslash i)) w(s)+\frac{1}{n}\left(v(N)-\sum_{j \in N} \frac{1}{\alpha} \sum_{S \ni j}(v(S)-v(S \backslash j)) w(s)\right) .
$$

Following the same reasoning as the one done in Proposition 1, we provide an explicit expression for the $I R L S^{w}$ value. Take $(N, v) \in \mathcal{G}$ and $A=\left\{j \in N: L S_{j}^{w}(N, v) \geq 0\right\}$. If $A=N$, then for every $i \in N$

$$
\operatorname{IRL} S_{i}^{w}(N, v)=L S_{i}^{w}(N, v)
$$

If $A \neq N$, then for every $i \in N$

$$
\operatorname{IRL} S_{i}^{w}(N, v)=\frac{1}{\alpha} \sum_{S \ni i}(v(S)-v(S \backslash i)) w(s)-\min \left\{\frac{1}{\alpha} \sum_{S \ni i}(v(S)-v(S \backslash i)) w(s), c\right\},
$$

where $c \geq 0$ is such that

$$
\sum_{j \in N} \min \left\{\frac{1}{\alpha} \sum_{S \ni j}(v(S)-v(S \backslash j)) w(s), c\right\}=\frac{1}{\alpha} \sum_{j \in N} \sum_{S \ni i}(v(S)-v(S \backslash i)) w(s)-v(N) .
$$

Finally, Ruiz et al. (1998b) pointed out that the additive normalization of a semivalue corresponds to a certain least square value. Hence, we can say that the truncated normalization of a semivalue corresponds to a certain individually rational least square value.

\section{Acknowledgements}

Authors acknowledge the financial support of Ministerio de Ciencia e Innovación through projects MTM2011-27731-C02 and MTM2011-27731-C03. Last but not least, we would like to thank the associated editor and the referees for their comments and suggestions which helped improve a previous version of the manuscript. Finally, the usual disclaimer applies.

\section{References}

Aumann, R. and Maschler, M. (1985). Game theoretic analysis of a bankruptcy problem from the Talmud. Journal of Economic Theory, 20:455-481.

Hammer, P. and Holzman, R. (1992). Approximations of pseudo-Boolean functions; applications to Game Theory. Zeitschrift für Operations Research, 36(1):3-21.

Owen, G. (1975). Multilinear extensions and the Banzhaf value. Naval Research Logistics Quarterly, 22:741-750. 
Ruiz, L. M., Valenciano, F., and Zarzuelo, J. M. (1996). The Least Square Prenucleolus and the Least Square Nucleolus. Two values for TU games based on the excess vector. International Journal of Game Theory, 25(1):113-134.

Ruiz, L. M., Valenciano, F., and Zarzuelo, J. M. (1998a). Some new results on Least Square Values for TU games. TOP, 6(1):139-158.

Ruiz, L. M., Valenciano, F., and Zarzuelo, J. M. (1998b). The family of Least Square Values for transferable utility games. Games and Economic Behavior, 24(1-2):109130. 